\title{
Atividade física associada ao ambiente urbano: um estudo com mulheres de três cidades litorâneas de Santa Catarina
}

http://dx.doi.org/10.11606/1807-5509201800020253

\author{
Vanessa Terezinha Ferrari JONCK ${ }^{*}$ \\ Camila da Cruz Ramos de ARAUJO* \\ Jorge Fernando HAMMES* \\ Joris PAZIN* \\ Leonessa BOING* \\ Mariana ROCHA* \\ Silvia Rosane PARCIAS* \\ Zenite MACHADO* \\ Adriana Coutinho de Azevedo GUIMARÃES*
}

*Centro de Ciências
da Saúde e do
Esporte, Universidade
do Estado de
Santa Catarina,
Florianópolis, SC,
Brasil.

\section{Resumo}

0 estudo analisou a prática de atividade física (AF) associada ao ambiente urbano de mulheres de cidades litorâneas de Santa Catarina (SC). Amostra foi composta por 1127 mulheres de Florianópolis, São José e Itajaí (SC). Utilizou-se o IPAQ - versão curta e o Questionário de Ambiente percebido: versão brasileira (NEWS). A coleta de dados ocorreu em agosto de 2012 a março de 2013. Realizou-se estatística descritiva, teste Qui quadrado, ANOVA com post hoc e análise de regressão multinomial bruta e ajustada $(p<0,05)$. Aproximadamente $50 \%$ das mulheres foram consideradas muito ativas, destacando-se Florianópolis, com maior tempo de AF moderada+vigorosa. Observou-se associação entre a prática de AF e presença de lixo próximo a sua casa, proximidade à orla, ruas iluminadas, convite de parentes e praça com equipamentos de ginástica. Concluiu-se que características do ambiente urbano parecem influenciar a prática de AF de mulheres muito ativas e ativas de três cidades litorâneas de SC.

Palavras-chave: Atividade motora; Saúde; Mulheres; Apoio social.

\section{Introdução}

A AF parece ser um dos fatores primordiais para melhorar a saúde da população, sendo também um importante recurso a ser utilizado na prevenção e controle de doenças ${ }^{1}$. Entretanto, ao longo dos últimos anos, a prevalência da AF tem sido associada às barreiras pessoais e aos fatores ambientais ${ }^{2}$.

As barreiras percebidas para a AF incluem tanto fatores internos (características individuais, menor prioridade para a $\mathrm{AF}$, envolvimento com atividades relacionadas à tecnologia) como fatores externos (influência da família e de outras pessoas, ausência de tempo, inacessibilidade e facilidades de custo) ${ }^{3}$. A literatura ainda aponta que características do ambiente como facilidade de acesso, disponibilidade de áreas verdes, espaços abertos para recreação, números de cruzamentos de ruas, segurança (geral e no trânsito), proximidade de locais, disponibilidade de calçadas e faixas de pedestres estáo associados com a prática de $\mathrm{AF}$, principalmente em pessoas adultas e idosas ${ }^{4-7}$.

A boa percepção de segurança, presença de academias, praças e quadras nos bairros, são algumas das variáveis que parecem influenciar positivamente a AF no lazer ${ }^{6}$. Entre norte-americanos, a insegurança nos bairros mostrou-se responsável pelo aumento de $191 \%$ na chance dos indivíduos em se tornarem inativos ${ }^{8}$. Em estudo realizado com homens e mulheres observou-se que $51 \%$ e $32 \%$, respectivamente, apresentavam menor probabilidade de atingirem alto nível de $\mathrm{AF}$ devido à insegurança? No caso específico das mulheres, aquelas que não se sentiam seguras à noite caminhavam menos do que aquelas que identificaram seus bairros como seguros?.

Noutro estudo com pessoas que possuem locais favoráveis à prática de AF públicos ou privados, próximos à residência, foi verificado que estes 
apresentam maior probabilidade de serem ativos no lazer ${ }^{10}$. O fato de se morar próximo a mercados, padarias, praças, entre outros, facilita e propicia deslocamentos a pé ou de bicicleta, confirmando os resultados de SALVADor et al. ${ }^{6}$ os quais relataram que as idosas consideradas mais ativas no tempo de lazer eram aquelas que realizavam caminhadas de 10 minutos para ir até igreja ou templo religioso.
Além disso, a presença de indivíduos realizando $\mathrm{AF}$ em praças e parques públicos pode contribuir para que outros usuários se sintam mais encorajados e incentivados à participar de atividades nestes locais ${ }^{4}$. Neste sentido, este estudo analisou a prática de $\mathrm{AF}$ associada às características do ambiente urbano em mulheres adultas de três cidades litorâneas de Santa Catarina.

\section{Método}

A amostra não probabilística por conveniência foi composta por mulheres de 20 a 59 anos de três cidades litorâneas de Santa Catarina, sendo estas, Florianópolis, São José e Itajaí. O critério de inclusão das cidades participantes privilegiou as cidades litorâneas com mais de 150 mil habitantes de acordo com o último Censo do Instituto Brasileiro de Geografia e Estatística (IBGE) realizado em 2010. Este estudo descritivo correlacional faz parte de um estudo mais amplo intitulado "Prevalência de atividade física de mulheres de Santa Catarina", o qual foi aprovado pelo Comitê de Ética - Pesquisa em Seres Humanos da Universidade Universidade do Estado de Santa Catarina em 02/03/12, protocolo no 214/2011.

Apesar de ser uma amostra não probabilística, foi realizado o cálculo do tamanho da amostra baseado na população de 297.545 mulheres de 20 a 59 anos, das cidades alvo, de acordo com o último censo do IBGE. O cálculo da amostra apresentou um erro de estimativa de $5 \%$ e intervalo de confiança de $95 \%$, sendo incluídas 1200 mulheres no estudo.

A coleta de dados ocorreu no período de agosto de 2012 a março de 2013 e as mulheres foram convidadas a participar do estudo voluntariamente. A fim de abranger um maior número da população de mulheres na faixa etária de 20 a 59 anos, as pesquisadoras entraram em contato com instituiçōes empregadoras ou prestadoras de serviços ao nível da educação, saúde, justiça, religião e seguros, além das donas de casa. Os questionários foram enviados por e-mail (encaminhado às participantes do estudo) e respondidos in loco com a presença das pesquisadoras. Foram distribuídos 1635 questionários (presenciais e on line), sendo que apenas 1127 foram devolvidos corretamente preenchidos. Quanto aos questionários impressos, 84 estavam preenchidos incorretamente, 97 voltaram em branco e 78 foram perdidos tanto no Correio quanto em lojas e empresas. Em paralelo, cinco questionários respondidos online estavam incompletos,
50 não chegaram aos destinatários, pois os endereços eletrônicos estavam incorretos e 194 não retornaram.

Para a coleta dos dados, foi utilizado um questionário autoaplicável, composto de instrumentos validados, dividido em quatro partes: a) Características pessoais: referente à idade, escolaridade, estado civil, número de filhos, cor da pele, peso e estatura (autorreferido) para cálculo do Índice de Massa Corporal - IMC (peso/estatura $^{2}$ ). Para classificação do IMC (status de peso) utilizou-se o protocolo da Organização Mundial de Saúde (2004) que estabelece os seguintes pontos de corte: abaixo do peso (IMC $<18,5$ ); normal (IMC 18,5-24,9); sobrepeso (IMC 25,0-29,9); sobrepeso e obesidade (IMC>30,0). Para fins estatísticos as categorias foram reagrupadas resultando em: peso normal: abaixo do peso e peso normal; acima do peso: sobrepeso e obesidade.

b) Estrato econômico: contendo o critério padrão de classificação econômica Brasil 2010 - Associação Brasileira de Empresas de Pesquisa (ABEP), considerado o principal instrumento de segmentaçáo da população segundo seu poder de compra, o qual classifica a população em classes econômicas A, B, $\mathrm{C}, \mathrm{D}$ e E, por meio da conversão da soma de pontos referentes ao grau de instrução da mulher pesquisada e a quantidade de bens de consumo existentes em sua residência ${ }^{11}$.

c) Nível de AF: A AF foi avaliada por meio do Questionário International Physical Activity Questionnaire (IPAQ - versão curta). Cada país participante adaptou e validou o questionário de acordo com as características da respectiva população. No Brasil, este questionário foi validado pelo Centro de Estudos do Laboratório de Aptidão Física de São Caetano do Sul - CELAFISCS, centro coordenador do IPAQ no Brasil ${ }^{12}$. Na avaliação da AF habitual por meio do IPAQ quantificou-se o número de vezes em que cada indivíduo realizou ao menos 10 minutos contínuos de caminhada, de AF 
de intensidade moderada e de intensidade vigorosa na última semana, em diversos envolvimentos, nomeadamente laboral, doméstico, lazer, recreativo e desportivo. Considerando-se que à caminhada é geralmente atribuído um nível de exigência de 3,3METs, ou seja, um nível superior ao valor de corte para a AF moderada (3METs), a AF total, que resulta do somatório da caminhada, da atividade moderada e da atividade vigorosa, corresponde a uma atividade de intensidade no mínimo moderada. A AF foi classificada em caminhada, AF moderada, moderada + vigorosa, vigorosa e total em minutos/dia. Foram consideradas muito ativas as participantes que realizaram atividade vigorosa maior ou igual a 3 vezes por semana com uma AF total de pelo menos $1500 \mathrm{MET}$-min/semana, ou qualquer AF (caminhada, moderada ou vigorosa) maior ou igual a 7 dias por semana com uma $\mathrm{AF}$ total de pelo menos $3000 \mathrm{MET}-\mathrm{min} / \mathrm{sem}$; foram consideradas ativas as participantes que realizaram AF moderada ou caminhada maior ou igual a 5 vezes por semana e maior ou igual a 30 minutos por dia, ou atividade vigorosa maior ou igual a 3 vezes por semana e maior ou igual a 20 minutos por dia, ou ainda qualquer AF (caminhada, moderada ou vigorosa) maior ou igual a 5 dias por semana com uma AF total de pelo menos $600 \mathrm{MET} / \mathrm{sem}$. Foram consideradas insuficientemente ativas as participantes que não cumpriram os requisitos necessários para serem consideradas ativas ou muito ativas e foram consideradas suficientemente ativas as participantes ativas e muito ativas. Foi igualmente estimado o tempo sentado.

d) Ambiente percebido: para a avaliação do ambiente percebido foi utilizada a versão adaptada da escala Neighborhood Environmental Walkability Scale (NEWS), para a atividade física a qual avalia as características do ambiente urbano (presença de locais para a prática de atividade física, segurança, características do ambiente percebido, oportunidades para a prática de atividade física e qualidade das ruas e calçadas e apoio social), adaptada por ReIs et al. ${ }^{13}$. Esta escala obteve resultados adequados de consistência interna, de validade e reprodutibilidade e já foi usada em estudos epidemiológicos com adultos e idosos na zona leste de São Paulo ${ }^{6,7}$. Considerado um questionário com respostas dicotômicas e interpretadas na forma de prevalência.

A primeira parte do questionário foi estruturada para que os sujeitos respondessem quanto tempo levariam caminhando para chegar das suas residências até diferentes pontos comerciais, de serviço ou lazer no bairro onde residiam. A segunda parte do questionário foi composta por questóes relacionadas às estruturas ambientais físicas e sociais (calçadas, aparência do bairro, vizinhos e amigos, presença de locais para praticar atividade física, segurança contra crimes e no trânsito, iluminação, esgoto, lixo, áreas verdes, etc.).

A análise estatística foi realizada por meio do Statistical Package for Social Sciences (SPSS) versão 20.0. Fez-se uso da estatística descritiva mediante o cálculo de média, desvio padrão e percentual. Para a comparação dos tipos/ intensidade de AF (caminhada, atividade física moderada e vigorosa) entre as cidades estudadas, foi utilizado a ANOVA seguida do teste Post Hoc de Bonferroni. A prevalência dos níveis de AF (insuficientemente ativo, ativo e muito ativo) foi expressa em percentagem. A comparação dos níveis de AF entre as três cidades e as características pessoais foi efetuada com o Teste Qui quadrado. Análise de regressão logística multinomial foi realizada para estimar razóes de chance bruta e ajustadas dos atributos do ambiente urbano e apoio social (variáveis independentes) associados a níveis de $\mathrm{AF}$ habitual. As associaçóes que apresentaram valor de $\mathrm{p} \leq 0,20$ na análise bivariada foram selecionadas para a entrada no modelo múltiplo ${ }^{14}$. Foi utilizada a estratégia forward selection para a modelagem. $\mathrm{O}$ nível de significância estatístico adotado nas análises de comparação entre grupos e de associaçóes entre variáveis foi de $\mathrm{p}<0,05$.

\section{Resultados}

Na TABELA 1 são apresentadas as características das participantes do estudo foram estratificadas de acordo com as cidades, nomeadamente o grupo 1 (Florianópolis), grupo 2 (São José) e o grupo 3 (Itajaí). A maioria das participantes do estudo tem ensino superior completo $(58,4 \%)$, mora com o companheiro $(55,3 \%)$, encontra-se com peso normal $(67,8 \%)$, apresenta uma percepção positiva em relação a sua saúde $(85,8 \%)$, pertence à classe econômica B $(69,1 \%)$ e está na faixa 
etária de 20 a 32 anos $(53,4 \%)$. Para todas estas variáveis houve diferença significativa $(\mathrm{p}=0,005$; $<0,001 ; \mathrm{p}<0,001 ; \mathrm{p}<0,001 ; \mathrm{p}<0,001 ; \mathrm{p}<0,001)$ respectivamente.

Em relaçáo à AF (TABELA 2) observa-se que as mulheres da cidade de Itajaí obtiveram a menor média na variável de AF vigorosa $(29 \pm 47 \mathrm{~min} / \mathrm{d})$, com diferença significativa $(\mathrm{p}<0,001)$. As mulheres de São José apresentaram as maiores médias no tempo que permanecem sentadas durante os dias de semana $(2025 \pm 1514 \mathrm{~min} / \mathrm{d})$ e finais de semana $(722 \pm 436 \mathrm{~min} / \mathrm{d})$, o que equivale a $\sim 7$ horas/dia e -6 horas/dia respectivamente, com diferença significativa em ambas as variáveis $(\mathrm{p}<0,001$; $\mathrm{p}<0,001)$. Já as mulheres da cidade de Florianópolis se sobressaíram na variável de AF classi ficada como moderada mais vigorosa $(113 \mathrm{~min} / \mathrm{d}) \mathrm{com}$ diferença significativa $(p=0,001)$. Entretanto a quantificaçấo da AF total não diverge entre as mulheres das cidades investigadas.
Quanto ao nível de AF (TABELA 3), foi verificado que a maioria das integrantes da amostra é considerada ativa ou muito ativa, destacando-se as provenientes de Florianópolis, cujo percentual de muito ativas é de $59,5 \%$. A diferença significativa verificada $(\mathrm{p}<0,001)$ evidencia que os grupos apresentam um nível de AF diferenciado.

O grupo de mulheres ativas (TABELA 4) apresentou menor chance para a prática de $\mathrm{AF}$ quando relatada presença de lixo próximo à residência $(\mathrm{p}=0,034)$.

No grupo de mulheres muito ativas (TABELA 5), observou-se que aquelas que moram próximo à orla têm maior chance de praticar AF ( $p=0,026)$, assim como aquelas que residem próximo a ruas bem iluminadas $(\mathrm{p}=0,045)$ e de praça com equipamentos $(p=0,047)$. Outro fator que parece ter influência na realização da prática de AF entre essas mulheres é o convite de parentes para a prática $(\mathrm{p}=0,001)$.
Teste do Qui quadrado $p<0,05$.
TABELA 1 - Características das participantes do estudo de acordo com as cidades. Florianópolis, São José e Itajaí - SC. 2012.

\begin{tabular}{|c|c|c|c|c|c|}
\hline Variáveis & Total & $\begin{array}{c}\text { Florianópolis } \\
\text { Grupo } 1\end{array}$ & $\begin{array}{l}\text { Sáo José } \\
\text { Grupo } 2\end{array}$ & $\begin{array}{c}\text { Itajaí } \\
\text { Grupo } 3\end{array}$ & p valor \\
\hline Escolaridade (\%) & & & & & $<0,001$ \\
\hline Ensino fundamental & 9,3 & 3,5 & 7,7 & 18,5 & \\
\hline Ensino médio & 32,3 & 14,8 & 56,3 & 24,9 & \\
\hline Ensino superior & 58,4 & 81,8 & 36,0 & 56,5 & \\
\hline Estado conjugal(\%) & & & & & 0,005 \\
\hline Morando junto & 55,3 & 58,7 & 48,6 & 58,9 & \\
\hline Morando separado & 44,7 & 41,3 & 51,44 & 41,1 & \\
\hline Estado nutricional (\%) & & & & & $<0,001$ \\
\hline Peso normal & 67,8 & 74,0 & 68,9 & 59,1 & \\
\hline Acima do peso & 32,2 & 26,0 & 31,1 & 40,9 & \\
\hline Estado de saúde(\%) & & & & & $<0,001$ \\
\hline Positivo & 85,8 & 91,5 & 81,5 & 84,1 & \\
\hline Negativo & 14,2 & 8,5 & 18,5 & 15,9 & \\
\hline Estrato econômico (\%) & & & & & $<0,001$ \\
\hline A & 12,2 & 18,0 & 10,8 & 7,1 & \\
\hline B & 69,1 & 74,5 & 73,8 & 57,4 & \\
\hline $\mathrm{C}$ & 17,6 & 7,5 & 14,9 & 32,5 & \\
\hline $\mathrm{D}$ & 1,1 & -- & 0,5 & 3,0 & \\
\hline Faixa etária(\%) & & & & & $<0,001$ \\
\hline 20 a 32 anos & 53,4 & 46,2 & 71,7 & 40,6 & \\
\hline 33 a 45 anos & 27,1 & 36,8 & 14,9 & 29,9 & \\
\hline 46 a 59 anos & 19,5 & 17,0 & 13,4 & 29,6 & \\
\hline
\end{tabular}


TABELA 2 - Caracterização da atividade física (média e desvio padrão) praticada pelas mulheres de acordo com as cidades. Florianópolis, São José e Itajaí - SC. 2012.

\begin{tabular}{lccccc}
\hline Variáveis & $\begin{array}{c}\text { Florianópolis } \\
\text { Grupo 1 }\end{array}$ & $\begin{array}{c}\text { Sáo José } \\
\text { Grupo 2 }\end{array}$ & $\begin{array}{c}\text { Itajaí } \\
\text { Grupo 3 }\end{array}$ & p valor & Post hoc \\
\hline Caminhada (min/d) & $45 \pm 47$ & $48 \pm 47$ & $51 \pm 51$ & 0,280 & --- \\
AF mod. (min/d) & $65 \pm 53$ & $59 \pm 62$ & $63 \pm 59$ & 0,334 & -- \\
AF vig. (min/d) & $48 \pm 53$ & $59 \pm 62$ & $29 \pm 47$ & $<0,001$ & $\mathrm{C}<\mathrm{A}, \mathrm{B}$ \\
AF mod+vig (min/d) & $113 \pm 88$ & $118 \pm 92$ & $92 \pm 84$ & 0,001 & $\mathrm{~A}>\mathrm{B}, \mathrm{C}$ \\
AF total & $159 \pm 112$ & $141 \pm 114$ & $143 \pm 109$ & 0,057 & --- \\
Sent./semana & $1574 \pm 946$ & $2025 \pm 1514$ & $1451 \pm 1100$ & $<0,001$ & $\mathrm{~B}>\mathrm{A}, \mathrm{C}$ \\
Sent./fds. & $624 \pm 401$ & $722 \pm 436$ & $559 \pm 445$ & $<0,001$ & $\mathrm{~B}>\mathrm{A}, \mathrm{C}$ \\
\hline
\end{tabular}

AF. Atividade física; mod. Moderada; vig. Vigorosa; min/d; minuto/ dia; Sentado/sem. Sentado semana; Sentado/fds. Sentado final de semana. Florianópolis $=A$; São José = B; Itajaí $=$ C; ANOVA; Post hoc Bonferroni $-p<0,05$

TABELA 3 - Nível de Atividade Física das participantes do estudo de acordo com as cidades. Florianópolis, São José e Itajaí - SC. 2012.

\begin{tabular}{lccccc}
\hline \multicolumn{1}{c}{ Variáveis } & Total & $\begin{array}{c}\text { Florianópolis } \\
\text { Grupo 1 }\end{array}$ & $\begin{array}{c}\text { Sáo José } \\
\text { Grupo 2 }\end{array}$ & $\begin{array}{c}\text { Itajaí } \\
\text { Grupo 3 }\end{array}$ & p valor \\
\hline Nível de AF (\%) & & & & & \\
Insuficientemente ativo & 26,6 & 20,5 & 31,6 & 28,1 & $<0,001$ \\
Ativo & 23,8 & 20,0 & 24,4 & 27,5 & \\
Muito ativo & 49,6 & 59,5 & 44,0 & 44,4 & \\
\hline
\end{tabular}

Nível de AF. Nível de atividade física. Teste do Qui quadrado $p<0,05$.

TABELA 4 - Razão de chance bruta e ajustada para nível de atividade física geral e ambiente físico construído e apoio social, entre mulheres ativas. Florianópolis, São José e Itajaí - SC. 2012.

\begin{tabular}{|c|c|c|c|}
\hline Variáveis & $\begin{array}{c}\# \\
\text { OR bruto (IC95\%) }\end{array}$ & ${ }^{*} \mathbf{p}$ & $\begin{array}{c}\# \\
\text { OR ajustado }{ }^{* * *}(\mathrm{IC} 95 \%)\end{array}$ \\
\hline \multicolumn{4}{|l|}{ Lixo próximo de casa" } \\
\hline $\begin{array}{l}\text { Sim } \\
\text { Não }\end{array}$ & $\begin{array}{c}0,69(0,48-0,98) \\
\text { Referência }\end{array}$ & 0,041 & $\begin{array}{c}0,37(0,16-0,84) \\
\text { Referência }\end{array}$ \\
\hline
\end{tabular}

TABELA 5 - Razão de chance bruta e ajustada para nível de atividade física geral e ambiente físico construído e ambiente social, entre mulheres muito ativas. Florianópolis, São José e Itajaí - SC. 2012.

\begin{tabular}{|c|c|c|c|c|}
\hline Variáveis & $\begin{array}{c}\# \\
\text { OR bruto (IC95\%) }\end{array}$ & ${ }^{*} \mathbf{p}$ & $\begin{array}{c}\# \\
\text { OR ajustado } \\
\text { *** } \\
(\text { IC95\%) }\end{array}$ & ${ }^{* *} \mathbf{p}$ \\
\hline \multicolumn{5}{|c|}{ Parque próximo de casa } \\
\hline $\begin{array}{l}\text { Sim } \\
\text { Náo }\end{array}$ & $\begin{array}{c}1,36(1,02-1,82) \\
\text { Referência }\end{array}$ & 0,034 & & \\
\hline \multicolumn{5}{|l|}{ Lugar para caminhar } \\
\hline $\begin{array}{l}\text { Sim } \\
\text { Não }\end{array}$ & $\begin{array}{c}1,36(1,02-1,81) \\
\text { Referência }\end{array}$ & 0,036 & & \\
\hline \multicolumn{5}{|l|}{ Quadra de esportes } \\
\hline $\begin{array}{l}\text { Sim } \\
\text { Não }\end{array}$ & $\begin{array}{c}1,37(1,04-1,83) \\
\text { Referência }\end{array}$ & 0,034 & & \\
\hline
\end{tabular}

- Local a até 10 minutos caminhando de casa. \# Categoria de referência utilizada: inativos ${ }^{* * *}$ As associações com $p \leq 0,20$ na análise bivariada foram selecionadas para a entrada no modelo múltiplo.

AF. Atividade física - Local a até 10 minutos caminhando de casa. \# Categoria de referência utilizada: inativos ${ }^{* * *}$ As associações com $p \leq 0,20$ na análise bivariada foram selecionadas para a entrada no modelo múltiplo. 
TABELA 5 - Razão de chance bruta e ajustada para nível de atividade física geral e ambiente físico construído e ambiente social, entre mulheres muito ativas. Florianópolis, São José e Itajaí - SC. 2012.

\begin{tabular}{|c|c|c|c|c|}
\hline Variáveis & $\begin{array}{c}\# \\
\text { OR bruto (IC95\%) }\end{array}$ & ${ }^{*} \mathbf{p}$ & $\begin{array}{c}\# \\
\text { OR ajustado *** (IC95\%) }\end{array}$ & ${ }^{* *} \mathbf{p}$ \\
\hline \multicolumn{5}{|l|}{ Campo de futebol } \\
\hline $\begin{array}{l}\text { Sim } \\
\text { Não }\end{array}$ & $\begin{array}{c}1,54(1,15-2,05) \\
\text { Referência }\end{array}$ & 0,040 & & \\
\hline \multicolumn{5}{|c|}{ Morar próximo a orla marítima } \\
\hline $\begin{array}{l}\text { Sim } \\
\text { Não }\end{array}$ & $\begin{array}{c}1,74(1,26-2,41) \\
\text { Referência }\end{array}$ & 0,001 & $\begin{array}{c}1,58(1,05-2,37) \\
\text { Referência }\end{array}$ & 0,026 \\
\hline \multicolumn{5}{|l|}{ Faixas de pedestre } \\
\hline $\begin{array}{l}\text { Sim } \\
\text { Não }\end{array}$ & $\begin{array}{c}1,43(1,03-1,98) \\
\text { Referência }\end{array}$ & 0,031 & & \\
\hline \multicolumn{5}{|l|}{ Ruas Bem iluminadas } \\
\hline $\begin{array}{l}\text { Sim } \\
\text { Não }\end{array}$ & $\begin{array}{c}1,46(1,08-1,97) \\
\text { Referência }\end{array}$ & 0,013 & $\begin{array}{c}1,41(1,10-1,98) \\
\text { Referência }\end{array}$ & 0,045 \\
\hline \multicolumn{5}{|c|}{ Amigo convida para fazer AF } \\
\hline $\begin{array}{l}\text { Sim } \\
\text { Não }\end{array}$ & $\begin{array}{c}1,48(1,10-2,00) \\
\text { Referência }\end{array}$ & 0,011 & & \\
\hline \multicolumn{5}{|c|}{ Parente convida para fazer $\mathrm{AF}$} \\
\hline $\begin{array}{l}\text { Sim } \\
\text { Não }\end{array}$ & $\begin{array}{c}1,62(1,20-2,18) \\
\text { Referência }\end{array}$ & 0,002 & $\begin{array}{c}1,64(1,10-2,67) \\
\text { Referência }\end{array}$ & 0,047 \\
\hline \multicolumn{5}{|c|}{ Praça com equipamentos próxima } \\
\hline $\begin{array}{l}\text { Sim } \\
\text { Náo }\end{array}$ & $\begin{array}{c}1,16(0,87-1,55) \\
\text { Referência }\end{array}$ & 0,311 & $\begin{array}{c}2,49(1,43-4,34) \\
\text { Referência }\end{array}$ & 0,001 \\
\hline
\end{tabular}

\section{Discussão}

A AF normalmente é influenciada por características individuais, bem como por fatores sociais e ambientais ${ }^{2}$. Níveis adequados de AF proporcionam benefícios para saúde física e mental ${ }^{15}$. O IPAQ é um dos instrumentos mais utilizados para estudos epidemiológicos brasileiros, apresentando vantagens em comparação a outros instrumentos, principalmente no que se refere à possibilidade de comparabilidade entre os estudos e na facilidade de aplicação em grandes amostras ${ }^{16}$.

As mulheres do presente estudo foram classificadas como suficientemente ativas (ativa + muito ativa) $(73,4 \%)$, com destaque para as residentes em Florianópolis (79,5\%). Essa maior prevalência de AF das mulheres de nosso estudo pode ser atribuído em parte, à localização das cidades pesquisadas, pois, acredita-se que indivíduos que residem no litoral são mais ativos (66,5\%) se comparados aos do interior $(53,4 \%)$ e áreas metropolitanas $(39,4 \%)^{17}$. Ainda, Giles-
Corti e Donovan ${ }^{18}$ apresentam como uma das instalações mais utilizadas para a prática de $\mathrm{AF}$ os espaços abertos públicos $(28,8 \%)$ e a praia $(22,7 \%)$.

As três cidades investigadas neste estudo são litorâneas. Acredita-se que o maior número de mulheres suficientemente ativas encontradas em Florianópolis deva-se ao fato de apresentar uma estrutura que estimula a prática de $\mathrm{AF}$ tanto em ambientes fechados como ao ar livre, além do desenvolvimento de diversos programas gratuitos de AF oferecidos em instituições de ensino superior e do próprio poder público municipal. Atrelados a este contexto, Florianópolis conta com duas vias planas e pavimentadas específicas para caminhada e corrida (aproximadamente $10 \mathrm{~km}$ de extensão de beira mar norte e $2,3 \mathrm{~km}$ de beira mar continental), 23 academias da saúde que foram projetadas para oferecer AF ao ar livre para adultos e idosos, 12 parques municipais que possibilitam a prática de $\mathrm{AF}$ por meio de trilhas ecológicas e caminhadas, sendo que alguns destes oferecem ainda áreas de lazer, ciclovias e 
quadras poliesportivas ${ }^{19}$. A maior prevalência de $\mathrm{AF}$ em Florianópolis pode estar relacionada também ao fato de que a cidade apresenta Índice de Desenvolvimento Humano (IDH) classificado como muito alto, sugerindo que existe uma relaçáo entre IDH e vida mais ativa ${ }^{20}$.

Quanto à intensidade da $\mathrm{AF}$ apresentada pelas mulheres deste estudo, pode-se observar que a atividade de intensidade moderada foi a mais prevalente nas três cidades, apesar de não haver diferença significativa entre as regióes estudadas. No entanto, verificaram-se diferenças na AF vigorosa e moderada+vigorosa ( $\mathrm{p}=0,001$ e $\mathrm{p}<0,001$, respectivamente). As mulheres da cidade de Itajaí apresentaram menor duração $(29 \pm 47 \mathrm{~min} / \mathrm{dia}) \mathrm{em}$ $\mathrm{AF}$ vigorosa e as mulheres de Florianópolis a maior duração em AF moderada+vigorosa (113 $\pm 88 \mathrm{~min} /$ dia). Resultados contrários foram encontrados por BAUMan et al. ${ }^{1}$, em que as mulheres de seis países dos 20 pesquisados, relataram maior prática de atividades moderadas. Neste contexto, a intensidade da AF constitui um aspecto determinante ${ }^{15}$ uma vez que a $A F$ de intensidade moderada e vigorosa está associada a maiores benefícios para a saúde ${ }^{21}$. Pessoas que praticam AF regularmente apresentam menor risco de desenvolver doenças cardiovasculares, diabetes, alguns tipos de câncer, obesidade, ansiedade e quadros de depressão, entre outras morbidades ${ }^{22}$.

As mulheres deste estudo, residentes em São José, apresentaram as maiores médias em relação ao tempo em que permanecem sentadas durante a semana e finais de semana, correspondendo a aproximadamente 8 e 6 horas, respectivamente. As mesmas médias foram encontradas num estudo realizado em estudantes universitários da regiáo Nordeste do Brasil ${ }^{23}$. Os resultados indicam que a maior prevalência de mulheres jovens (72\%) encontra-se na cidade de São José. Talvez isso possa esclarecer o fato destas mulheres apresentarem as maiores médias em relação ao tempo em que permanecem sentadas, pois, acredita-se que mulheres mais jovens estáo consolidando suas carreiras, ou seja, passam um tempo maior sentadas trabalhando e/ou estudando, dispondo de menor tempo para a prática de $\mathrm{AF}^{24}$. Evidencia-se que a falta de tempo e a jornada de trabalho ou de estudos são motivos frequentemente apontados como principais empecilhos para adesão à prática de $\mathrm{AF}$, entre indivíduos de diversas faixas etárias e categorias profissionais ${ }^{25}$. Além disso, os estudantes estão continuamente sujeitos a trabalhos, prazos de entrega, longas horas de estudo e situaçóes de exaustáo física e mental ${ }^{23}$.

Percebe-se que a presença de lixo próximo à residência foi identificada como barreira associada à prática de $\mathrm{AF}$ em mulheres ativas das três cidades litorâneas de Santa Catarina, incluídas no presente estudo. Barreiras semelhantes foram encontradas no bairro Ermelino Matarazzo, localizado na zona leste de São Paulo, no qual ruas com poluição e esgotos a céu aberto foram correlacionadas negativamente com a prática de $\mathrm{AF}$ no lazer de adultos ${ }^{26}$. A proximidade a orla marítima, característica predominante nas três cidades, pareceu apresentar influência na prática de AF das mulheres muito ativas, fator este já observado em estudo feito em Florianópolis, com indivíduos de ambos os sexos ${ }^{4}$. Florianópolis é considerada uma cidade que apresenta estaçóes climáticas bem definidas, com uma temperatura média anual bastante agradável ${ }^{5}$, o que parece influenciar a prática de AF nas proximidades a orla marítima. Ainda, McComarck et al. ${ }^{27}$ revelam que áreas com uma melhor estética e cenário agradável são associadas positivamente com a prática de AF. No presente estudo, as ruas bem iluminadas também foram relacionadas com a prática de $\mathrm{AF}$, sendo este fator intimamente ligado com a percepção de segurança do local para a prática, citada em outros estudos ${ }^{4}$.

O fator motivacional identificado como "convite de parentes" foi relacionado positivamente com a prática de $\mathrm{AF}$ entre as mulheres muito ativas. Essa mesma relação foi observada por FLORINDo et al. ${ }^{7}$ e GIEHL et al. ${ }^{5}$ ao investigarem a prática de AF na população de adultos e idosos, respectivamente. Segundo Salvador et al. ${ }^{6}$, essa prática torna-se mais agradável quando se tem companhia, e o suporte social atua intrinsecamente por meio do incentivo e de parcerias e, extrinsecamente, motivando pessoas a se exercitarem no ambiente das cidades.

Além disso, praças com equipamentos também revelaram influência nesta prática, como no estudo de Salvador et al. ${ }^{6}$ em que idosas que residiam próximo a praças tiveram aproximadamente quatro vezes mais chances de serem ativas no lazer. Esses espaços urbanos que oferecem estruturas de lazer para a prática de AF de fácil acesso podem desempenhar um papel importante nos padróes de comportamento ativo da populaçãos.

Como na maioria dos estudos sobre AF, a mesma foi avaliada por meio de um questionário, o que representa uma limitaçáo para esse tipo de estudo, no entanto, recomendaçóes para a atividade de melhoria da saúde física são, em sua maioria, com 
base em estudos epidemiológicos que utilizaram a mesma metodologia. Outra limitação do estudo está relacionada à seleção da amostra, por tratar-se de uma amostra não probabilística por conveniência, apresentou-se heterogênea em alguns aspectos, como escolaridade, estrato econômico e faixa etária.

Algumas características do ambiente urbano, como ausência de lixo próximo a residência, residir próxima a orla marítima, ter acesso a ruas iluminadas e praças com equipamentos de ginástica, e o apoio social, como o convite de parentes, parecem influenciar a prática de AF das mulheres muito ativas e ativas de três cidades litorâneas de Santa Catarina. Apesar das limitaçôes relacionadas ao instrumento, os resultados do estudo poderão contribuir para o avanço científico nesta temática, além de permitir comparaçôes e novas reflexôes em outros contextos.

\section{Abstract \\ Physical activity associated to urban environment: a study with women from three coast cities of Santa Catarina}

The study aimed to analyze the practice of physical activity (PA) associated with the urban environment of women from coastal cities of Santa Catarina (SC). Sample consisted of 1127 women from Florianópolis, São José and Itajai (SC). We used the IPAQ - short version and noticed Environment Questionnaire: Brazilian version (NEWS). Data collection occurred in August 2012 to March 2013. Performed descriptive statistics, Chi square test, ANOVA and post hoc analysis of crude and adjusted $(p<0.05)$ multinomial regression. Approximately $50 \%$ of women were considered very active, especially Florianópolis, which showed longer moderate + vigorous PA. We observed an association between the practice of AF and presence of garbage near his home, near the waterfront, lighted streets, invite relatives and square with fitness equipment. It was concluded that urban environment characteristics seem to influence the practice of PA very active and active women from three coastal cities of SC.

KEYWORDS: Motor activity; Health; Women; Social support.

\section{Referências}

1. Bauman A, Bull F, Chey T, Craig CL, Ainsworth BE, Sallis JF, et al. The International Prevalence Study on Physical Activity: results from 20 different countries. Int J Behav Nutr Phys Act. 2009;6(89):21-32.

2. Bolivar J, Daponte A, Rodríguez M, Sánchez JJ. The influence of individual, social and physical environment factors on physical activity in the adult population in Andalusia, Spain. Int J Environ Res Public Health. 2010;7:60-77.

3. Allison KR, Dwyer JJ, Goldenberg E, Fein A, Yoshida KK, Boutilier M. Male adolescents' reasons for participating in physical activity, barriers to participation, and suggestions for increasing participation. Adolescence. 2005;40(157):155-70.

4. Pazin J, Duarte MFS, Borgatto AF, Peres MA. Ambiente urbano percebido e apoio social para a atividade física no lazer e no deslocamento em adultos de Florianópolis, SC. Rev Bras Ativ Fís Saúde. 2012;17(2):100-6.

5. Giehl MWC, Schneider IJC, Corseuil HX, Benedetti TRB, D’Orsi E. Atividade física e percepção do ambiente em idosos: estudo populacional em Florianópolis. Rev Saúde Pública. 2012;46(3):516-25.

6. Salvador EP, Florindo AA, Reis RS, Costa EF. Percepção do ambiente e prática de atividade física no lazer entre idosos. Rev Saúde Pública. 2009;43(6):972-80.

7. Florindo AA, Salvador EP, Reis RS, Guimarães VV. Percepção do ambiente e prática de atividade física em adultos residentes em região de baixo nível socioeconômico. Rev Saúde Pública. 2011;45(2):302-10.

8. Boehmer TK, Lovegreen SL, Haire-Joshu D, Brownson RC. What constitutes an obesogenic environment in rural communities?. Am J Health Promot. 2006;20(6):411-21.

9. Bennet GG, Mcneill LH, Wolin KY, Duncan DT, Puleo E, Emmons KM. Safe to walk? Neighborhood safety and physical activity among public housing residents. PLoS Med. 2007;4(10):1559-607. 
10. Florindo AA, Hallal PC, Moura EC, Malta DC. Prática de atividades físicas e fatores associados em adultos. Rev Saúde Pública. 2009;43:65-73.

11. Associação Brasileira de Empresas de Pesquisa. Critério de Classificação Econômica Brasil. São Paulo; 2010.

12. Pardini R, Matsudo S, Matsudo TAV, Andrade E, Braggion G, Andrade D, et al. Validation of the international physical activity questionnaire (IPAQ): pilot study in brazilian young adults. Med Sci Sports Exerc. 1997;29:S5-S9.

13. Reis MS, Reis RS, Hallal PC. Validade e fidedignidade de uma escala de avaliação do apoio social para a atividade física. Rev. Saúde Pública. 2011;45(2):294-301.

14. Hosmer DM, Lemeshow S. Applied logistic regression. New York: John Wiley \& Sons; 1989.

15. Haskell WL, Lee I-M, Pate RR, Powell KE, Blair SN, Franklin BA, et al. Physical activity and public health: updated recommendation for adults from the American College of Sports Medicine and the American Heart Association. Med Sci Sports Exerc. 2007;39(8): 1423-34.

16. Hallal PC, Dumith SC, Bastos JP, Reichert FF, Siqueira FV, Azevedo MR. Evolução da pesquisa epidemiológica em atividade física no Brasil: revisão sistemática. Rev. Saúde Pública. 2007;41(3):453-60.

17. Matsudo SM, Matsudo VR, Araújo T, Andrade D, Andrade E, Oliveira L, et al. Nível de atividade física da população do estado de São Paulo: análise de acordo com o gênero, idade, nível sócio econômico, distribuição geográfica e de conhecimento. RBCM. 2002;10(4):41-50.

18. Giles-Corti B, Donovan RJ. The relative influence of individual, social and physical environment determinants of physical activity. Soc Sci Med. 2002;54(12):1793-812.

19. Prefeitura Municipal de Florianópolis [Internet]. Florianópolis [citado 2013 out. 17]. Disponível em: http://www.pmf.sc.gov.br/.

20. Organização das Nações Unidas, Instituto de Pesquisa e Economia Aplicada, Fundaçáo João Pinheiro. Atlas do Desenvolvimento Humano no Brasil [Internet]. Brasília; 2013. [citado 2013 out. 17]. Disponível em: http://atlasbrasil.org.br/2013/.

21. Brown DW, Brown DR, Heath GW, Balluz L, Giles WH, Ford ES, et al. Associations between physical activity dose and health-related quality of life. Med Sci Sports Exerc. 2004;36:809-96.

22. Mok Y, Won S, Kimm H, Nam C, Ohrr H, Jee SH. Physical activity level and risk of death: the severance cohort study. J Epidemiol. 2012;22(6):494-500

23. Fontes ACD, Vianna RPT. Prevalência e fatores associados ao baixo nível de atividade física entre estudantes universitários de uma universidade pública da regiáo Nordeste - Brasil. Rev Bras Epidemiol. 2009;12(1):20-9.

24. Sousa TFD, Fonseca AS, Barbosa AR. Perceived barriers by university students in relation the leisure-time physical activity. Rev Bras Cineantropom Desempenho Humano. 2013;15(2):164-73.

25. Castro-Carvajal JA, Patiño-Villada FA, Cardona-Rendón BM, Ochoa-Patiño V. Aspectos asociados a la actividad física en el tiempo libre en la población adulta de un municipio antioqueño. Rev Salud Pública. 2008;10(5):679-90.

26. Florindo AA, Garcia LMT, Guimarães VV, Salvador EP, Fonseca FH, Reis RS, et al. Escore de ambiente construído relacionado com a prática de atividade física no lazer: aplicação numa regiáo de baixo nível socioeconômico. Rev Bras Cineantropom Desempenho Hum. 2013;15(2):243-55.

27. McCormack G, Giles-Coiti B, Lange A, Smith T, Martin K, Pikora, TJ. An update of recent evidence of the relationship between objective and seK-report measures of the physical environment and physical activity behaviours. J Sci Med Sport. 2004;7(1):S81-S92.

\begin{tabular}{r|r} 
ENDEREÇo & \\
Leonesa Boing & \\
Centro de Ciências da Saúde e do Esporte & Recebido para publicação: 08/o6/2014 \\
Universidade do Estado de Santa Catarina & Revisado: 18/04/2016 \\
R. Pascoal Simone, 358 & Aceito: 28/03/2018 \\
88080-350 - Florianópolis - SC - BRASIL & \\
leonessaboing@gmail.com &
\end{tabular}


